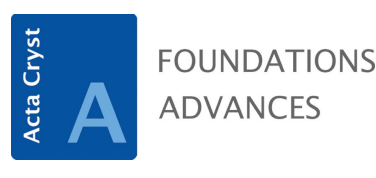

ISSN 2053-2733

Received 28 June 2019

Accepted 3 September 2019

Edited by L. D. Marks, Northwestern University, USA

Keywords: electron diffraction; atomic scattering factors; relativity theory; Schrödinger equation.

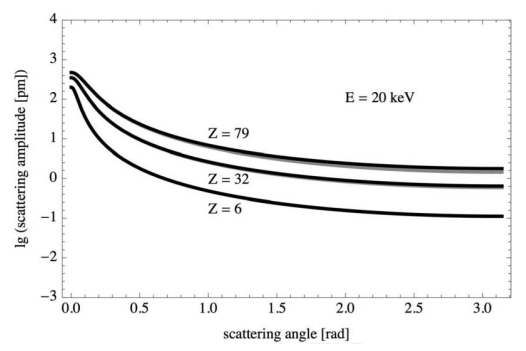

OPEN $\odot$ ACCESS

\section{Relativistic correction of atomic scattering factors for high-energy electron diffraction}

\author{
Markus Lentzen* \\ Ernst Ruska Centre, Forschungszentrum Jülich $\mathrm{GmbH}, 52425$ Jülich, Germany. ${ }^{*}$ Correspondence e-mail: \\ m.lentzen@fz-juelich.de
}

Relativistic electron diffraction depends on linear and quadratic terms in the electric potential, the latter being neglected in the frequently used relativistically corrected Schrödinger equation. The quadratic electric potential term modifies atomic scattering amplitudes in particular for large-angle scattering and backscattering. The respective correction increases with increasing scattering angle, increasing atomic number and increasing kinetic energy. Conventional tabulations for electron scattering and its large-angle extrapolations can be amended in closed form by a universal correction based on the screened Coulomb potential squared.

\section{Introduction}

A frequently used framework for the calculation of highenergy electron diffraction by an atom or ion is the solution of the relativistically corrected Schrödinger equation (Molière, 1947; Fujiwara, 1961) with a model for the atomic or ionic electric potential. These model potentials are tabulated for a wide range of atomic numbers and frequently occurring ionic charges in the form of scattering factors (Doyle \& Turner, 1968; Doyle \& Cowley, 1974; Rez et al., 1994, 1997) or their parameterizations (Doyle \& Turner, 1968; Doyle \& Cowley, 1974; Fox et al., 1989; Rez et al., 1994, 1997; Waasmaier \& Kirfel, 1995; Weickenmeier \& Kohl, 1998; Peng, 1998; Lobato \& Van Dyck, 2014); see Kirkland (2010) for a survey. Conventionally, tables of the scattering factors display the Born scattering amplitude, that is the Fourier transform of the electric potential times an interaction constant. A relativistic correction, dependent on the electron speed, is applied to the tabulated values, which can be directly used to determine scattering cross sections on the first Born approximation.

The normal form of the relativistically corrected Schrödinger equation (Molière, 1947; Fujiwara, 1961) is linear in the electric potential, yet the correct relativistic energymomentum relation, which is the basis of the Klein-Gordon equation (Klein, 1926; Gordon, 1926; Kragh, 1984), contains an additional quadratic term in the electric potential. That term is neglected in the above conventional framework, and thus, to the best of our knowledge, no tabulations exist for fully corrected relativistic scattering factors.

The aim of this work is to explore the impact of the quadratic electric potential term on atomic or ionic electron scattering amplitudes in particular at large angles, including backscattering. Furthermore, a method is proposed to amend the existing tables for the Born scattering factors. The work presents a brief survey of the required theory, calculations for a set of atoms of small, medium and large atomic number at 
small, medium and large electron energy, and concludes with a discussion of possible applications.

\section{Theory}

\subsection{Wave equations}

The relativistic energy-momentum relation (Einstein, 1905)

$$
\left(E_{0}+E+V\right)^{2}-p^{2} c^{2}=E_{0}^{2}
$$

with rest energy $E_{0}=m c^{2}$, kinetic energy $E$ in vacuum, potential energy $V=e \phi$, momentum $p$, speed of light $c$, rest mass $m$, elementary charge $e$ and electric potential $\phi$ is divided by $2\left(E_{0}+E\right)$ and rearranged:

$$
\frac{p^{2}}{2 m^{*}}=E^{*}+V+\frac{\beta^{2} V^{2}}{4 E^{*}} .
$$

Thus the quadratic energy relation (1) adopts a form akin to a linear energy relation with the parameters $m^{*}=\gamma m, \beta=v / c$, electron speed $v$,

$$
\gamma=\frac{1}{\left(1-\beta^{2}\right)^{1 / 2}}=1+\frac{E}{E_{0}},
$$

and

$$
E^{*}=\frac{1}{2} m^{*} v^{2}
$$

the relativistically modified kinetic energy in vacuum.

The Klein-Gordon equation (Klein, 1926; Gordon, 1926; Kragh, 1984) for fixed kinetic energy is derived by substituting the momentum operator $-i \hbar \nabla$ for the momentum $p$, and the relativistically corrected Schrödinger equation (Molière, 1947; Fujiwara, 1961) by further neglecting the squared potential term. As usual, $h$ denotes the Planck constant $h$ divided by $2 \pi$.

The scattering amplitude $f(\mathbf{g})$ is derived from the wave equations by an ansatz for the wavefunction,

$$
\psi(\mathbf{r})=\exp (2 \pi i \mathbf{k} \cdot \mathbf{r})+f(\mathbf{g}) \frac{\exp (2 \pi i k r)}{r},
$$

which describes the scattering of a plane wave with wavevector $\mathbf{k}$ into a spherical wave with an amplitude dependent on the scattering vector $\mathbf{g}$. The wavevector of a plane partial wave after scattering is thus $\mathbf{k}+\mathbf{g}$, and $\mathbf{r}$ denotes a coordinate in real space. For elastic scattering $\mathbf{k}$ and $\mathbf{k}+\mathbf{g}$ are equal in magnitude,

$$
g=2 k \sin \frac{\theta}{2}
$$

and

$$
k=\frac{1}{\lambda}=\frac{\left(\gamma^{2}-1\right)^{1 / 2}}{\lambda_{\mathrm{C}}},
$$

with $\theta$ the scattering angle between $\mathbf{k}$ and $\mathbf{k}+\mathbf{g}$, and $\lambda_{\mathrm{C}}$ the Compton wavelength.

The amplitude of the spherical wave is determined in the far field, at large distance $r$ from a scattering region bounded by a sphere of diameter $d$, with $k r \gg d^{2}$. If the bounded region contains a single atom, the scattering amplitude is called the atomic form factor. The far-field solution of the wave equation in the above sense is found on the first Born approximation (Born, 1926) to the first order of an effective potential $V_{\text {eff }}$, with the well-known result:

$$
f(\mathbf{g})=\frac{2 \pi m^{*}}{h^{2}} \iiint V_{\mathrm{eff}}(\mathbf{r}) \exp (-2 \pi i \mathbf{g} \cdot \mathbf{r}) \mathrm{d} \mathbf{r} .
$$

For the relativistically corrected Schrödinger equation $V_{\text {eff }}=$ $V$, and for the Klein-Gordon equation $V_{\text {eff }}=V+\beta^{2} V^{2} / 4 E^{*}$. The tabulations of atomic form factors according to equation (8) are used in two ways. The first, and obvious, is the display of the atomic scattering amplitude, its modulus squared being the differential scattering cross section (see the next section). The second is the indirect, through the Fourier transform in equation (8), but exact display of the atomic scattering potential.

\subsection{Scattering amplitudes for a screened Coulomb potential}

The integral (8) for the scattering amplitude of a screened atomic Coulomb potential (Wentzel, 1926)

$$
V=\frac{Z E_{\mathrm{h}} a_{0}}{r} \exp (-r / R)
$$

with atomic number $Z$, Hartree energy $E_{\mathrm{h}}$, Bohr radius $a_{0}$ and screening radius (Lenz, 1954)

$$
R=\frac{1}{2 \pi g_{0}}=\frac{a_{0}}{Z^{1 / 3}}
$$

can be solved in closed form, with the well-known result (Wentzel, 1926):

$$
f_{1}(\mathbf{g})=\frac{\gamma Z}{2 \pi^{2} a_{0}\left(g^{2}+g_{0}^{2}\right)} .
$$

The scattering amplitude of the squared atomic Coulomb potential term

$$
\frac{\beta^{2} V^{2}}{4 E^{*}}=\frac{\beta^{2} Z^{2} E_{\mathrm{h}}^{2} a_{0}^{2}}{4 E^{*} r^{2}} \exp (-2 r / R)
$$

can be found in closed form as well:

$$
f_{2}(\mathbf{g})=\frac{Z^{2} \alpha^{2}}{2 \pi g} \arctan \frac{g}{2 g_{0}},
$$

with the fine structure constant $\alpha$.

Both $f_{1}(\mathbf{g})$ and $f_{2}(\mathbf{g})$ have a maximum at $\mathbf{g}=\mathbf{0}$,

$$
f_{1}(\mathbf{0})=\frac{\gamma Z}{2 \pi^{2} a_{0} g_{0}^{2}}=2 \gamma a_{0} Z^{1 / 3}
$$

and

$$
f_{2}(\mathbf{0})=\frac{Z^{2} \alpha^{2}}{4 \pi g_{0}}=\frac{1}{2} \alpha^{2} a_{0} Z^{5 / 3},
$$

and for small scattering vectors $f_{1}(\mathbf{g})$ is always much larger than $f_{2}(\mathbf{g})$. A comparison of the asymptotes for large scattering vectors,

$$
f_{1} \rightarrow \frac{\gamma Z}{2 \pi^{2} a_{0} g^{2}}
$$


(Rutherford, 1911) and

$$
f_{2} \rightarrow \frac{Z^{2} \alpha^{2}}{4 g},
$$

reveals that $f_{2}(\mathbf{g})$ can become equal in magnitude to $f_{1}(\mathbf{g})$ for large $Z$. This is particularly clear for the asymptotic values of backscattering

$$
f_{1} \rightarrow \frac{Z \alpha^{2} a_{0}}{2 \gamma \beta^{2}}
$$

and

$$
f_{2} \rightarrow \frac{\pi Z^{2} \alpha^{3} a_{0}}{4 \gamma \beta},
$$

when $\theta=\pi$ and $g$ adopts the largest possible value $2 k$. The contribution $f_{2}$ to the total scattering amplitude $f=f_{1}+f_{2}$ becomes significant for large-angle scattering and backscattering.

The differential cross section is

$$
\mathrm{d} \sigma=|f|^{2} \mathrm{~d} \Omega=2 \pi|f(\theta)|^{2} \sin \theta \mathrm{d} \theta
$$

for scattering into a solid angle $\mathrm{d} \Omega$ and azimuthal symmetry.

\section{Calculation of scattering amplitudes and cross sections}

Born scattering amplitudes [equation (8)] were calculated for carbon $(Z=6)$, germanium $(Z=32)$ and gold $(Z=79)$ at kinetic energies of 20,200 and $2000 \mathrm{keV}$ over the full range of scattering angles, $\theta=0 \ldots \pi$. Two different models were used for the scattering potential: the screened Coulomb potential [equation (9)] and the screened Coulomb potential extended by the squared Coulomb potential term [equation (12)]. The scattering amplitudes for both models, $f_{1}$ and $f_{1}+f_{2}$, are displayed in Figs. 1, 2 and 3. The difference between the two scattering amplitudes increases with increasing scattering angle, increasing atomic number and increasing kinetic energy.

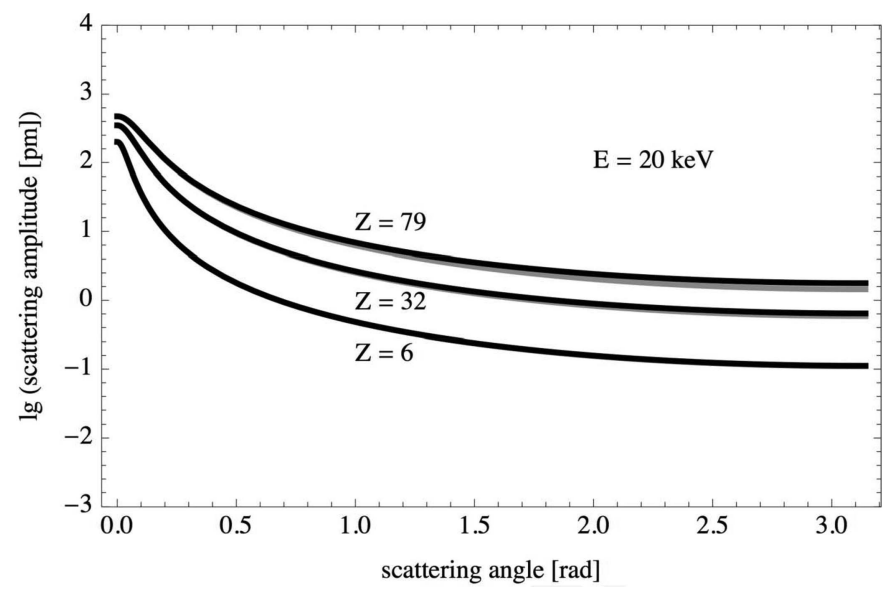

Figure 1

Born scattering amplitudes $f_{1}$ (grey) and $f_{1}+f_{2}$ (black) on a logarithmic scale versus scattering angle for carbon, germanium and gold at a kinetic energy of $20 \mathrm{keV}$.
The difference between the two models can be further expressed by calculating Born scattering cross sections for total scattering, $\theta=0 \ldots \pi$, which denotes the total mismatch. The relative difference between the two models is pronounced for large scattering angles, and thus it is instructive to further calculate the cross section for backscattering, $\theta=\pi / 2 \ldots \pi$. A third important measure is the cross section for scattering outside the typical acceptance angle of an electron microscope, $\theta=250 \mathrm{mrad} \ldots \pi$.

The respective cross sections on the two models, and the relative differences, are compiled in Tables 1,2 and 3, again for carbon $(Z=6)$, germanium $(Z=32)$ and gold $(Z=79)$ at kinetic energies of 20,200 and $2000 \mathrm{keV}$. The relative differences of the total cross sections decrease for increasing energy; they increase for backscattering and scattering outside the microscope acceptance angle with increasing energy. With increasing atomic number the relative differences increase in any category.

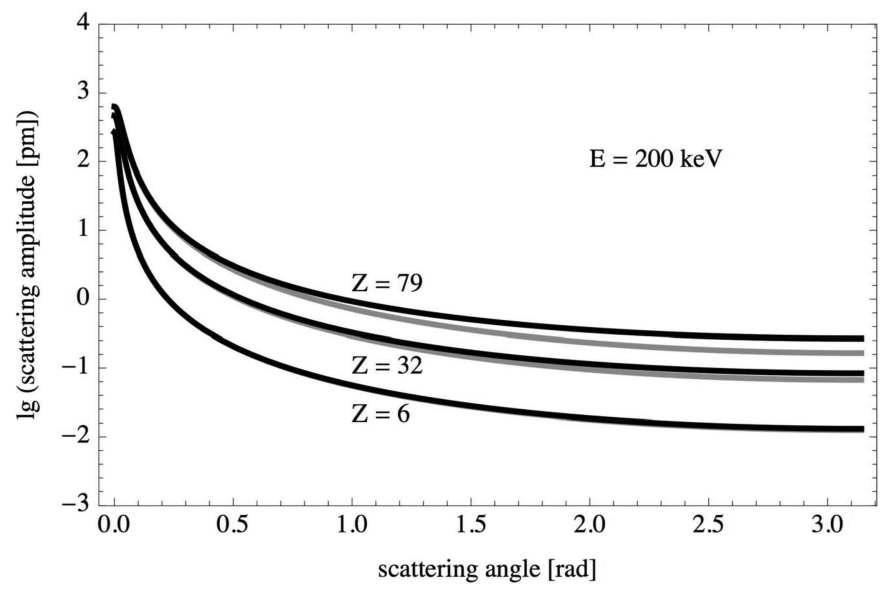

Figure 2

Born scattering amplitudes $f_{1}$ (grey) and $f_{1}+f_{2}$ (black) on a logarithmic scale versus scattering angle for carbon, germanium and gold at a kinetic energy of $200 \mathrm{keV}$.

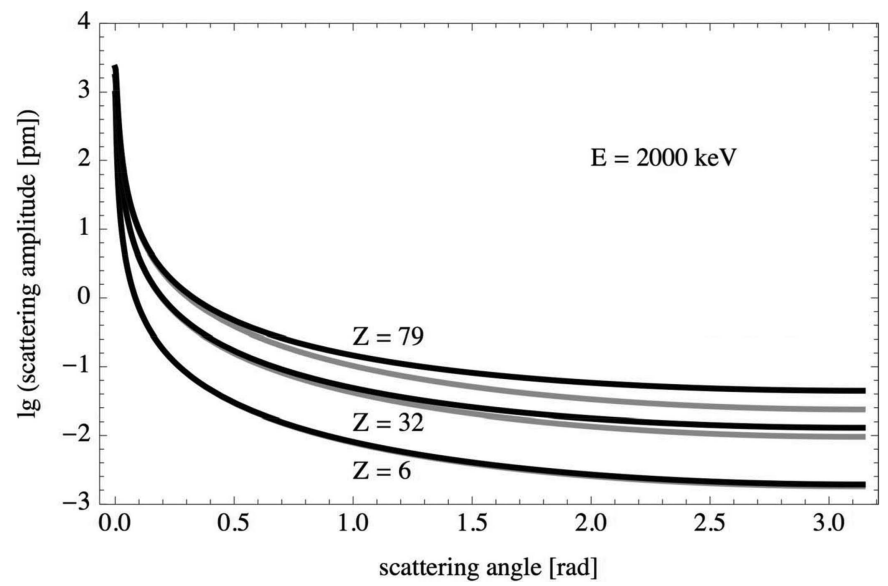

Figure 3

Born scattering amplitudes $f_{1}$ (grey) and $f_{1}+f_{2}$ (black) on a logarithmic scale versus scattering angle for carbon, germanium and gold at a kinetic energy of $2000 \mathrm{keV}$. 
Table 1

Born scattering cross sections $\sigma\left(\mathrm{pm}^{2}\right)$ and relative errors for carbon, for various scattering angles and kinetic energies.

\begin{tabular}{llll}
\hline$\theta$ & Total & $>250 \mathrm{mrad}$ & $>\pi / 2$ \\
\hline $20 \mathrm{keV}$ & & & \\
$\sigma\left(f_{1}\right)$ & 276.3 & 9.31 & 0.152 \\
$\sigma\left(f_{1}+f_{2}\right)$ & 276.5 & 9.38 & 0.157 \\
$200 \mathrm{keV}$ & $0.10 \%$ & $0.73 \%$ & $2.92 \%$ \\
$\sigma\left(f_{1}\right)$ & & & \\
$\sigma\left(f_{1}+f_{2}\right)$ & 42.26 & 0.125 & 0.00198 \\
$2000 \mathrm{keV}$ & 42.29 & 0.128 & 0.00214 \\
$\sigma\left(f_{1}\right)$ & $0.07 \%$ & $2.01 \%$ & $7.40 \%$ \\
$\sigma\left(f_{1}+f_{2}\right)$ & & & \\
& 21.31 & 0.00256 & 0.0000405 \\
& 21.32 & 0.00264 & 0.0000451 \\
\hline
\end{tabular}

\section{Discussion}

Conventional tables of the scattering factors $f_{1}$ (Doyle \& Turner, 1968; Doyle \& Cowley, 1974; Rez et al., 1994, 1997; Kirkland, 2010) are organized such that the Born scattering amplitude [equation (8)] is only tabulated for a range of scattering vectors where Rutherford scattering is modified by the effects of screening, up to, e.g., $s=g / 2=60.0 \mathrm{~nm}^{-1}$. The amplitudes for larger scattering vectors are understood to be calculated with the Rutherford formula [equation (16)]. In a last step the tabulated values have to be multiplied by $\gamma$ as the interaction constant used in the tabulations conventionally contains $m$ and not $m^{*}$.

The above standard procedure can be amended to include the effects of the squared potential term [equation (12)], thus providing a proper relativistic correction. Although $f_{2}$ was calculated for the screened Coulomb potential in equation (13), it can serve as a universal correction, because in the range of small scattering vectors, where the details of the screening would play a role, $f_{2}$ is dominated by $f_{1}$. For larger scattering vectors, in the regime of Rutherford scattering [equation (16)], $f_{2}$ has the proper asymptote [equation (17)].

Thus the conventional tables can be used to derive the proper relativistic scattering amplitude:

(i) Multiply tabulated values by $\gamma$.

(ii) Extrapolate the tabulated range through the Rutherford formula [equation (16)]. (10).

(iii) Determine the screening parameter $g_{0}$ using equation

(iv) Add the squared potential term $f_{2}$ using equation (13).

Once the scattering amplitude is determined, a Fourier transform to real space provides the effective potential to be used in diffraction calculations on the Klein-Gordon equation. The above treatment of the squared potential term allows, however, the use of simpler algorithms for the solution of the Schrödinger equation instead.

The implementation of the proper relativistic scattering amplitude is particularly suitable for the phase grating approximation of the multislice algorithm (Cowley \& Moodie, 1957). The calculation of the phase grating requires a projection of the potential along the chief propagation direction, which is achieved by evaluating the structure factor
Table 2

Born scattering cross sections $\sigma\left(\mathrm{pm}^{2}\right)$ and relative errors for germanium, for various scattering angles and kinetic energies.

\begin{tabular}{llll}
\hline$\theta$ & Total & $>250 \mathrm{mrad}$ & $>\pi / 2$ \\
\hline $20 \mathrm{keV}$ & & & \\
$\sigma\left(f_{1}\right)$ & 2571.4 & 247.1 & 4.31 \\
$\sigma\left(f_{1}+f_{2}\right)$ & 2593.9 & 256.3 & 5.00 \\
$200 \mathrm{keV}$ & $0.87 \%$ & $3.61 \%$ & $13.9 \%$ \\
$\sigma\left(f_{1}\right)$ & & & \\
$\sigma\left(f_{1}+f_{2}\right)$ & 393.7 & 3.54 & 0.0564 \\
$2000 \mathrm{keV}$ & 396.4 & 3.93 & 0.0823 \\
$\sigma\left(f_{1}\right)$ & $0.68 \%$ & $9.90 \%$ & $31.4 \%$ \\
$\sigma\left(f_{1}+f_{2}\right)$ & & & \\
& 198.6 & 0.0729 & 0.00115 \\
\hline
\end{tabular}

Table 3

Born scattering cross sections $\sigma\left(\mathrm{pm}^{2}\right)$ and relative errors for gold, for various scattering angles and kinetic energies.

\begin{tabular}{llll}
\hline$\theta$ & Total & $>250 \mathrm{mrad}$ & $>\pi / 2$ \\
\hline $20 \mathrm{keV}$ & & & \\
$\sigma\left(f_{1}\right)$ & 8567.8 & 1391.6 & 26.15 \\
$\sigma\left(f_{1}+f_{2}\right)$ & 8815.1 & 1518.4 & 36.89 \\
$200 \mathrm{keV}$ & $2.80 \%$ & $8.35 \%$ & $29.1 \%$ \\
$\sigma\left(f_{1}\right)$ & & & \\
$\sigma\left(f_{1}+f_{2}\right)$ & 1313.5 & 21.42 & 0.344 \\
$2000 \mathrm{keV}$ & 1343.9 & 27.41 & 0.784 \\
$\sigma\left(f_{1}\right)$ & $2.26 \%$ & $21.8 \%$ & $56.1 \%$ \\
$\sigma\left(f_{1}+f_{2}\right)$ & & & \\
& 662.6 & 0.444 & 0.00702 \\
\hline
\end{tabular}

of an atomic arrangement with the component of the scattering vector along the propagation direction set to zero. Including the squared potential term would now involve a Fourier transform to real space, calculation of the squared potential and line integrations along the propagation direction, or alternatively a numerically costly convolution in reciprocal space. Compared with the latter, the prescription given in this work provides a numerically very efficient way to determine the respective additional structure factor based on the form factors $f_{2}$.

The squared potential correction [equation (13)] is obviously most significant for backscattering, as can be deduced from the scattering cross sections displayed in the rightmost columns of Tables 1,2 and 3. The error by neglecting the correction can be as large as $66.7 \%$ for the case of gold at a kinetic energy of $2 \mathrm{MeV}$. The modification of backscattering cross sections extends, however, into the region of medium electron energies and medium to small charge numbers. A striking example is the cross section for knock-on damage in germanium, which involves scattering angles from $2.5 \mathrm{rad}$ to $\pi$ for a kinetic energy of $400 \mathrm{keV}$ to transfer the required displacement energy of $15 \mathrm{eV}$ to a germanium atom. The Born cross section for this process is $0.00316 \mathrm{pm}^{2}$, but only $0.00189 \mathrm{pm}^{2}$ by neglecting correction (13), which is a difference of $40.2 \%$. An example of knock-on damage of a light element is oxygen displacement in magnesium oxide at a 
displacement energy of $55 \mathrm{eV}$. The Born cross section for this process at $400 \mathrm{keV}$ electron energy is $0.000504 \mathrm{pm}^{2}$, but only $0.000441 \mathrm{pm}^{2}$ by neglecting correction (13), which is a difference of $12.5 \%$.

The modification of the cross sections for scattering outside the acceptance angle of an electron microscope indicates that there is also an impact on an important parameter of forward scattering, namely electron absorption. In transmission electron microscopy the bore of the objective pole-piece limits the cone of scattered electrons to a semi-angle of around $250 \mathrm{mrad}$, and thus a certain fraction of scattered intensity is missing in the image plane underneath; it appears to be absorbed by the imaging system. For larger kinetic energies and larger atomic numbers the estimate of that apparent electron absorption would be in error on the linear model $f_{1}$ alone.

\section{Conclusion}

The conventional framework of electron scattering by an electric potential is modified by an additional quadratic term in the electric potential, if the correct relativistic energymomentum relation (1) is considered. The respective modification of atomic scattering amplitudes increases with increasing scattering angle, increasing atomic number and increasing kinetic energy. Conventional tabulations for electron scattering (Doyle \& Turner, 1968; Doyle \& Cowley, 1974; Rez et al., 1994, 1997; Kirkland, 2010) and its large-angle extrapolations can be amended in closed form by a universal correction [equation (13)] based on the screened Coulomb potential squared [equation (12)].

\section{References}

Born, M. (1926). Z. Phys. 38, 803-827.

Cowley, J. M. \& Moodie, A. F. (1957). Acta Cryst. 10, 609-619.

Doyle, P. A. \& Cowley, J. M. (1974). International Tables for X-ray Crystallography, Vol. IV, pp. 152-175. Birmingham: Kynoch Press.

Doyle, P. A. \& Turner, P. S. (1968). Acta Cryst. A24, 390-397.

Einstein, A. (1905). Ann. Phys. 322, 891-921.

Fox, A. G., O'Keefe, M. A. \& Tabbernor, M. A. (1989). Acta Cryst. A45, 786-793.

Fujiwara, K. (1961). J. Phys. Soc. Jpn, 16, 2226-2238.

Gordon, W. (1926). Z. Phys. 40, 117-133.

Kirkland, E. J. (2010). Advanced Computing in Electron Microscopy, 2nd ed. New York: Springer.

Klein, O. (1926). Z. Phys. 37, 895-906.

Kragh, H. (1984). Am. J. Phys. 52, 1024-1033.

Lenz, F. (1954). Z. Naturforsch. Teil A, 9, 185-204.

Lobato, I. \& Van Dyck, D. (2014). Acta Cryst. A70, 636-649.

Molière, G. (1947). Z. Naturforsch. Teil A, 2, 133-145.

Peng, L.-M. (1998). Acta Cryst. A54, 481-485.

Rez, D., Rez, P. \& Grant, I. (1994). Acta Cryst. A50, 481-497.

Rez, D., Rez, P. \& Grant, I. (1997). Acta Cryst. A53, 522.

Rutherford, E. (1911). London Edinb. Dubl. Philos. Mag. J. Sci. 21, 669-688.

Waasmaier, D. \& Kirfel, A. (1995). Acta Cryst. A51, 416-431.

Weickenmeier, A. L. \& Kohl, H. (1998). Acta Cryst. A54, 283-289.

Wentzel, G. (1926). Z. Phys. 40, 590-593. 